\title{
An evaluation of genotyping by sequencing (GBS) to map the Breviaristatum-e (ari-e) locus in cultivated barley
}

Hui Liu ${ }^{1,4+}$, Micha Bayer ${ }^{1 \dagger}$, Arnis Druka ${ }^{1}$, Joanne R Russell ${ }^{1}$, Christine A Hackett ${ }^{2}$, Jesse Poland ${ }^{3}$, Luke Ramsay ${ }^{1}$, Pete E Hedley ${ }^{1}$ and Robbie Waugh ${ }^{1,5^{*}}$

\begin{abstract}
We explored the use of genotyping by sequencing (GBS) on a recombinant inbred line population (GPMx) derived from a cross between the two-rowed barley cultivar 'Golden Promise' (ari-e.GP/Vrs 1) and the six-rowed cultivar 'Morex' (Ari-e/vrs1) to map plant height. We identified three Quantitative Trait Loci (QTL), the first in a region encompassing the spike architecture gene Vrs 1 on chromosome $2 \mathrm{H}$, the second in an uncharacterised centromeric region on chromosome $3 \mathrm{H}$, and the third in a region of chromosome $5 \mathrm{H}$ coinciding with the previously described dwarfing gene Breviaristatum-e (Ari-e).

Background: Barley cultivars in North-western Europe largely contain either of two dwarfing genes; Denso on chromosome 3H, a presumed ortholog of the rice green revolution gene OsSd1, or Breviaristatum-e (ari-e) on chromosome $5 \mathrm{H}$. A recessive mutant allele of the latter gene, ari-e.GP, was introduced into cultivation via the $\mathrm{cV}$. 'Golden Promise' that was a favourite of the Scottish malt whisky industry for many years and is still used in agriculture today.
\end{abstract}

Results: Using GBS mapping data and phenotypic measurements we show that ari-e.GP maps to a small genetic interval on chromosome $5 \mathrm{H}$ and that alternative alleles at a region encompassing $\mathrm{Vrs} 1$ on $2 \mathrm{H}$ along with a region on chromosome $3 \mathrm{H}$ also influence plant height. The location of Ari-e is supported by analysis of near-isogenic lines containing different ari-e alleles. We explored use of the GBS to populate the region with sequence contigs from the recently released physically and genetically integrated barley genome sequence assembly as a step towards Ari-e gene identification.

Conclusions: GBS was an effective and relatively low-cost approach to rapidly construct a genetic map of the GPMx population that was suitable for genetic analysis of row type and height traits, allowing us to precisely position ari-e.GP on chromosome 5H. Mapping resolution was lower than we anticipated. We found the GBS data more complex to analyse than other data types but it did directly provide linked SNP markers for subsequent higher resolution genetic analysis.

Keywords: Barley, Dwarfing gene, Genotyping by sequencing, Physical map

\footnotetext{
* Correspondence: robbie.waugh@hutton.ac.uk

${ }^{\dagger}$ Equal contributors

'Cell and Molecular Sciences, The James Hutton Institute, Invergowrie,

Dundee, Scotland DD2 5DA, UK

${ }^{5}$ Division of Plant Sciences, James Hutton Institute, Invergowrie, The

University of Dundee. College of Life Sciences, Dundee, Scotland DD2 5DA,

UK

Full list of author information is available at the end of the article
}

\section{Biomed Central}

(c) 2014 Liu et al.; licensee BioMed Central Ltd. This is an Open Access article distributed under the terms of the Creative Commons Attribution License (http://creativecommons.org/licenses/by/2.0), which permits unrestricted use, distribution, and reproduction in any medium, provided the original work is properly cited. The Creative Commons Public Domain Dedication waiver (http://creativecommons.org/publicdomain/zero/1.0/) applies to the data made available in this article, unless otherwise stated. 


\section{Background}

Barley (Hordeum vulgare L.) is a diploid $(2 \mathrm{n}=14)$ economically important cereal crop and genetic model for small grain temperate cereals. Golden Promise (GP) is a two-rowed UK spring barley cultivar, and is currently the most responsive genotype for barley genetic transformation. Also, because of its unique properties, the malt extracted from GP is used to distil a number of signature Single Malt Scotch whiskies such as Macallan and Glengoyne. It is a primary induced gamma-ray mutant derivative of the barley cultivar Maythorpe, and is known to contain a mutation in Breviaristatum-e (Ari-e). This mutation in Ari-e in GP (ari-e.GP, also referred to in the literature as GP erectoides) causes a semidwarfing phenotype that has been used widely in barley cultivar development (especially in Scotland) to shorten straw length and reduce the severity of lodging. GP is also susceptible to several fungal pathogens, has short awns (as well as being dwarf), reduced internode length and shows a measure of tolerance to salt [1]. Genetic analysis has previously located ari-e.GP to barley chromosome $5 \mathrm{H}$ as a quantitative trait locus (QTL) influencing plant height, and physiological studies have confirmed its relative insensitivity to the addition of exogenous gibberellic acid $\left(\mathrm{GA}_{3}\right)$ [2]. The Ari-e gene has not yet been cloned although it was recently mapped as a height QTL using the tools of contemporary biometrical genetics in a complex three-way cross [3].

Over the past two decades, many molecular tools have been developed in barley to enable genetic research [4-9]. The primary focus has been the construction of molecular marker-based genetic linkage maps that can be leveraged for mapping genes of interest and subsequent marker assisted selection in breeding programs. These have been applied to discover, dissect and manipulate genes determining a range of simple and complex traits. Because of their value, accompanied by their increasing use in genetics and breeding, there has been a continual drive to both reduce marker costs and to avoid ascertainment issues [10] while at the same time enhancing flexibility and marker throughput per assay. It is therefore appropriate that new developments in marker technology are both explored and thoroughly evaluated against the current state of the art. Now that next generation sequencing (NGS) technology has been shown to be capable of discovering and genotyping thousands of markers across almost any genome of interest at low cost and in a single step, a current debate is whether sequence-based genotyping methods are ready to replace many of the established and widely used tools such as highly-multiplex Single Nucleotide Polymorphism (SNP) platforms [9].

Available sequence-based genotyping methods generally rely upon the use of restriction enzymes to produce a reduced representation of the non-repetitive (low copy) regions of the genome. Restriction site-associated genomic DNA (RAD) typing is such an approach and has been used in several species for the construction of linkage maps and application in QTL analyses [11]. In barley, a RAD linkage map was recently produced in a double haploid population and used for QTL analysis [12]. Elshire and colleagues [13] subsequently described a similar but more straightforward method of genotyping by sequencing (GBS) which works effectively in 96-well (or higher) plate assays. GBS was originally developed for high-resolution association studies in maize [14] and, like RAD, has been extended to a range of species with complex genomes. A two-enzyme GBS protocol has now been developed that produces a uniform library for sequencing and has been applied to both wheat and barley [15]. This GBS approach has been shown to be suited to genetic analysis of rapeseed, lupin, lettuce, switchgrass, soybean, and maize [16-20].

In this report, our biological objective was to identify at high resolution the genetic location of the ari-e.GP semi-dwarfing gene of cultivated barley. However, as a sequence assembly of the barley genome has just been published [21,22], we also wanted to use a sequence-based genetic marker methodology that would in principle allow us to link directly to the genome sequence assemblies and physical map, ultimately as a shortcut to facilitate the identification of the Ari-e gene. We therefore chose to explore use of the two-enzyme based GBS method, using digestion of genomic DNA with a six-base methylation sensitive 'rare-cutter' and a four-base 'common cutter' enzyme. We combined this with the Illumina NGS platform and developed a downstream informatics pipeline to discover co-dominant (SNPs) in an $\mathrm{F}_{11}$ single-seed descent mapping population from a Golden Promise (GP) by Morex $(\mathrm{Mx})$ cross. In contrast to GP, Mx is a tall spring six-rowed North American barley variety with desirable malting and brewing characteristics. Most importantly, $\mathrm{Mx}$ is the reference cultivar used in the barley genome sequencing efforts. Our genetic analysis using GBS data from the recombinant inbred (RIL) population confirmed the location of ari-e.GP on barley chromosome $5 \mathrm{H}$. In the process we discovered 1,949 high-confidence SNPs that we could associate with contigs in the NGS sequence assemblies and physical map.

\section{Results and discussion}

\section{Golden Promise by Morex population (GPMx) and} variation in plant height

A recombinant inbred line (RIL) population of $160 \mathrm{~F}_{11}$ single-seed descent lines from a GP by Mx cross was developed over seven years, from 2003-2012, at the James Hutton Institute. The $136 \mathrm{~F}_{11}$ RILs used in this study comprised 56 two-rowed and 80 six-rowed accessions. 
The population segregates quantitatively for height as shown in Figure 1 varying between different RILs from 60 to $130 \mathrm{~cm}$. Asymmetric transgressive segregation of plant height across the GPMx population can be observed - there were around 40 lines taller than Morex, but only about 10 shorter than Golden Promise. The Pearson correlation between the heights in the two years was 0.887 . Two lines showed a marked difference in height between the two years.

\section{Generation of Pstl reference sequences from barley genome assemblies}

To facilitate genetic analysis by GBS we first extracted a set of $64 \mathrm{bp}$ reference sequences flanking all predicted Pst I restriction sites from barley genome assemblies of the cultivars Morex (genome coverage: (53X) [22]), Bowman (26X) and Barke (20X) using the 'restrict' program from the EMBOSS suite of tools (see Methods). For cultivar Morex, 343,854 restriction sites were identified, yielding a total of 633,331 GBS reference sequences present on 251,433 unique Morex genome assembly contigs. Of all the identified sites, 54,377 flanking sequences had to be excluded because the restriction site was too close to the start or end of an assembled genomic contig and therefore extraction of the full 64 bp sequence was impossible. Extraction of additional sequences unique to the genome assemblies of cultivars Bowman and Barke yielded a further 71,519 and 97,764 sequences, respectively. After removal of chloroplast (cp) sequences a total of 802,046 reference sequences remained that were subsequently used for read mapping. More than half (54\%) of the 64 bp reference sequences stemmed from Morex contigs that contain regions of homology to full length cDNAs or expressed genes, previously mapped genetic markers $(\mathrm{cM})$ or sequences that have chromosome arm assignments based on survey sequencing of flow sorted chromosome arms [23].

\section{GBS reads of GPMx}

We generated three 48-plex GPMx GBS libraries (GPMx_1-3) representing all 136 progenies and the parents, which were repeatedly represented in each library for QC purposes. We used PstI combined with MseI to digest genomic DNA, with the PstI overhang sequence located in the barcode adapter adjacent to the barcode sequence, and the MseI overhang sequence located in common Y-adapter [15] (barcode sequences in Additional file 1: Table S1). Single-end sequencing starting from the barcoded adapter was performed using Illumina chemistry. Pilot sequencing the GPMx_1 library on an Illumina GAII platform generated more than 61 million single-end reads of $72 \mathrm{bp}$ in length. Of these, over $58 \mathrm{M}$ reads were categorised as having a correct barcode and PstI overhang sequence (from here we call this proportion of the sequences 'categorised reads'). Further sequencing of all three GPMx population libraries, each on one lane of an Illumina HiSeq2000, generated a total of $622 \mathrm{M}$ reads of $100 \mathrm{bp}$ and more than $482 \mathrm{M}$ remaining as categorised reads (see criteria below). The average number of categorised reads obtained per lane was $28.5 \mathrm{M}$ on the Illumina GA II and $160 \mathrm{M}$ on the Illumina HiSeq2000. By applying various filtering criteria (i.e. presence of accurate barcode and complete PstI overhang sequence, and no undetermined nucleotides (Ns) in the reads), the percentages of categorised reads were $93.4 \%$ (Illumina GA II) and

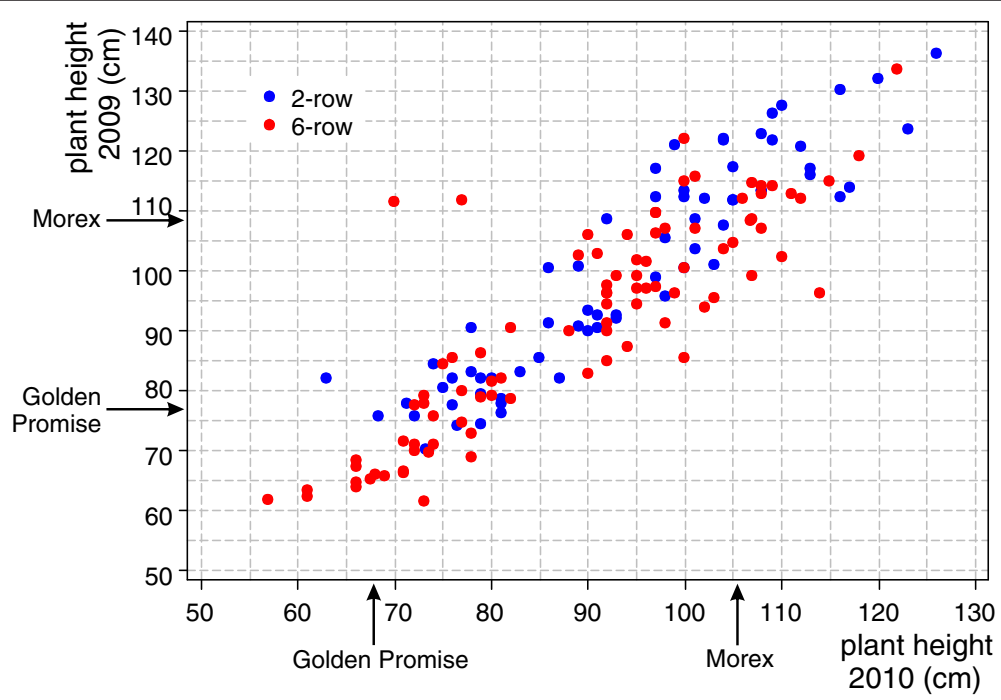

Figure 1 Scatterplot of plant height measurements of individual GPMx lines collected from the plants grown in polytunnel (in 2009, $\mathrm{Y}$-axis) and in the field (in 2010, $\mathrm{X}$-axis). Two-rowed lines are shown in blue and six-rowed in red. 
77.5\% (Illumina Hiseq2000). After deconvolution the distribution of the number of reads per sample ranged from 520,427 to $6,554,933$ (Additional file 2: Table S2). The read distribution was relatively even across the population, with only 3 of the 138 lines having less than a million reads. For the parents, we obtained $8.2 \mathrm{M}$ reads from Golden Promise and 11.5 M from Morex, due to repeat sequencing. All sequence reads generated from GPMx were submitted to the Sequence Read Archive section of the European Nucleotide Archive (ENA) (submission: ERP002594 Genotyping by sequencing of a barley mapping population).

Co-dominant markers from the GPMx population datasets In total, $461 \mathrm{M}$ categorized reads from the GPMx mapping population were mapped to the $64 \mathrm{bp}$ reference sequences using the Bowtie mapping tool [24]. In order to reduce the number of false positive SNPs during downstream analysis, only a single mismatch per read was allowed, and only uniquely mapped reads were included, leaving $46 \%$ of $461 \mathrm{M}$ reads mapped to the reference. These categorized reads were then evaluated for single base-pair differences across the population. We removed all dominant markers from the dataset because of our inability to distinguish null alleles from missing data. Using these highly conservative criteria, we identified an initial set of 1,949 co-dominant SNPs with robust allele calls across the population.

\section{Linkage mapping of GPMx population}

The 1,949 codominant SNPs were analysed using JoinMap. They were first checked for identical pairs based on segregation data across the population and on this basis 267 were excluded, always dropping the SNP marker with the lower quality score in each identical pair. A further 291 SNPs were excluded as they had more than $20 \%$ missing values. The remaining 1,391 high confidence SNPs were clustered into seven linkage groups with the number of markers per group ranging from 109 to 270, with nine remaining isolated at a LOD of six. These linkage groups were ordered using JoinMap's maximum likelihood mapping algorithm. SNPs with a poor fit to the neighbouring SNPs were excluded and the linkage analysis was rerun leaving a total of 1,332 unique high quality SNPs incorporated into the map. The numbers of co-dominant GBS SNPs are presented in Table 1.

\section{Location of SIX ROWED SPIKE 1}

A major developmental gene, SIX ROWED SPIKE 1 (VRS1), segregates in the GPMx population. The VRS1 gene has previously been identified [25] and profoundly affects barley spike morphology, but its effect on other plant traits such as height in GPMx is not known. Barley plants carrying a recessive vrs1 allele (e.g. vrs1.a Morex) develop spikes containing six rows of grain in contrast to the ancestral wild type spike which develops only two rows (e.g. Vrs1.b Golden Promise). Alternative alleles at VRS1 also influence the number of tillers that develop on a plant and could, as suggested previously, affect plant height, and this would influence our subsequent analysis of ari-e.GP. The most significant associations between row type and SNPs were with MR_2568613P909R13 and MR_57812P2860R48. Both mapped to $80.5 \mathrm{cM}$ on chromosome $2 \mathrm{H}$ (Figure 2A). All 80 six-rowed lines had the same genotype as Morex, while the 56 two-rowed lines had the same genotype as Golden Promise (i.e. there were no recombinants between these markers and VRS1).

\section{A major plant height QTL overlaps with the Breviaristatum-e (Ari-e) locus}

We mapped plant height as a quantitative trait for each year separately using the GPMx GBS linkage map. A permutation test with 1,000 permutations had a 95th percentile of 3.0 for each year's height data, and this was used as a genome-wide LOD threshold. This resulted in the identification of three significant plant height QTLs on chromosomes $2 \mathrm{H}, 3 \mathrm{H}$ and $5 \mathrm{H}$ (Figure 2). The major plant height QTL was located on chromosome $5 \mathrm{H}$ (Figure 2C). For each year's height data, the SNP most closely associated with height was MR_47526P1793R57 at 29.7 cM on chromosome $5 \mathrm{H}$. This SNP explained $55.2 \%$ of the variance in height in 2009, with the 'bb' genotype having a mean height 27.5 (SE 2.1) cm higher than the 'aa' genotype, and $61.6 \%$ of the variance in height in 2010, with the 'bb' genotype having a mean height 24.8 (SE 1.7) cm higher than the 'aa' genotype.

Previously, it was shown that Golden Promise carries a mutation in the dwarfing gene known as Breviaristratum-e (Ari-e) [26]. The position of the gene has been roughly estimated as about $30 \mathrm{cM}$ from the $S H O R T$ RACHILLA HAIR (srh) locus [27]. Two induced mutant alleles of Ari-e (ari-e.GP (Golden Promise) and ari-e.1 (cv. 'Bonus')), were introgressed as $\mathrm{BC}_{6} \mathrm{~F}_{3}$ lines into the

Table 1 Number of GPMx SNPs mapped

\begin{tabular}{|c|c|c|c|c|c|c|c|c|}
\hline Chromosome & $1 \mathrm{H}$ & $2 \mathrm{H}$ & $3 \mathrm{H}$ & $4 \mathrm{H}$ & $5 \mathrm{H}$ & $6 \mathrm{H}$ & 7H & Total \\
\hline SNPs clustering & 151 & 270 & 195 & 109 & 187 & 203 & 267 & 1,382 \\
\hline SNPs mapped & 150 & 259 & 176 & 97 & 187 & 201 & 262 & 1,332 \\
\hline Additional cosegregating SNPs & 20 & 60 & 45 & 12 & 19 & 51 & 57 & 264 \\
\hline
\end{tabular}


A

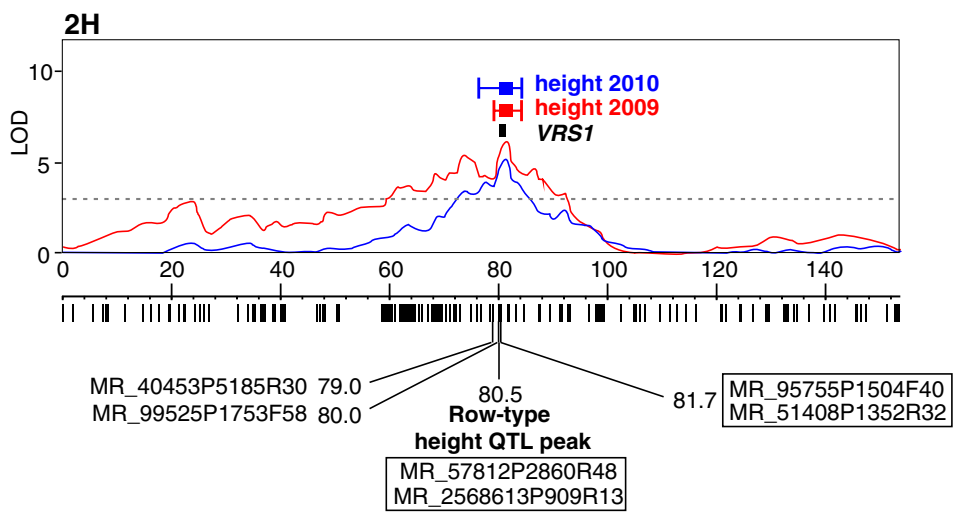

$\mathrm{B}$

3H

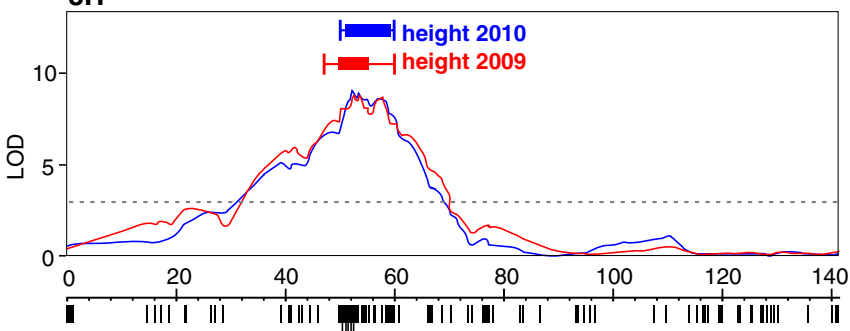

BK_1629470P50F30 51.0 (

BK_343652P3505R6 51.2 52.7

MR_159225P1301R26 51.

height QTL peak

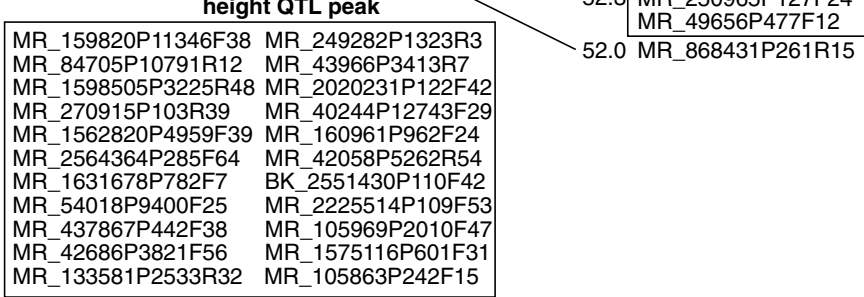

C

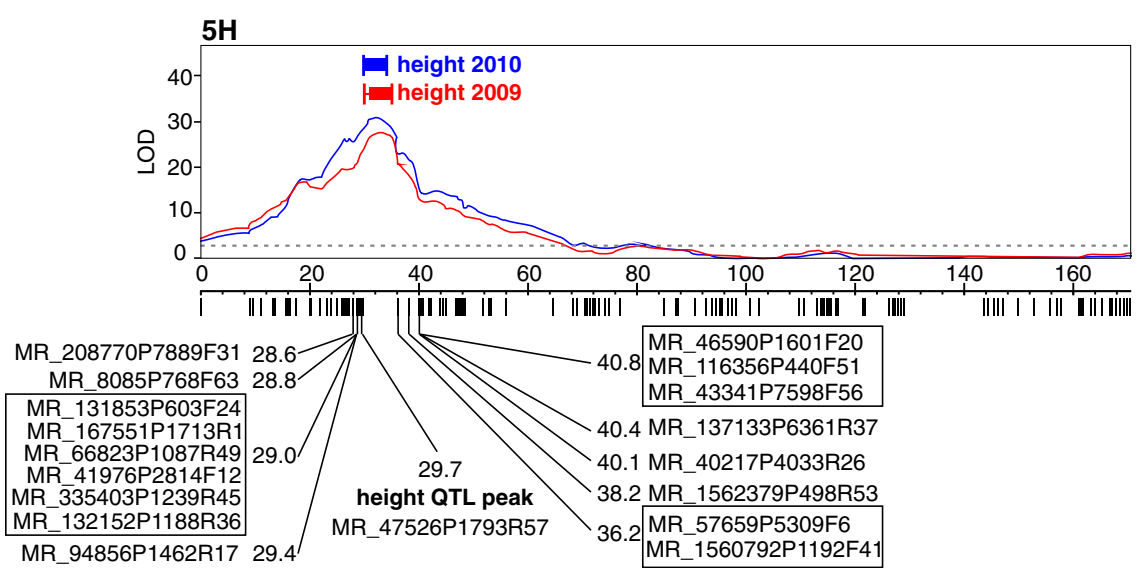

Figure 2 QTL LOD profiles of plant height and the position of VRS1, determined using 138 GPMx RILs. Only chromosomes with significant QTLs are shown: $2 \mathrm{H}$ on panel $\mathbf{A}, 3 \mathrm{H}$ on panel $\mathbf{B}$ and $5 \mathrm{H}$ on panel $\mathbf{C}$. Vertical lines below the graphs show markers on the GBS map. Marker names and GBS map positions surrounding relevant loci are also shown. Co-segregating markers are outlined. The 2009 data are shown in red and 2010 data are in blue. The horizontal dotted line shows the significance threshold. Boxes show the one-LOD support intervals, and whiskers show the two-LOD intervals. 
background of cv. 'Bowman' resulting in lines BW042 and BW043 [28]. Cross-referencing the SNP markers that define the introgressed region in BW043 (ari-e.GP), which has a genetically well-defined introgression, with the barley genome sequence assembly [22] supports ari-e. GP as the gene underlying the plant height QTL identified using the GPMx population. This also supports the early observation of Ari-e being linked to srh [27], as BW873, a nearly isogenic line of $\mathrm{cv}$. Bowman carrying srh, contains an introgressed segment located 10-30 cM distal to the GPMx height QTL [28].

Restricted multiple QTL mapping (rMQM) detected two further QTLs for height, the most significant markers being MR_1631678P782F7 at 51.6 cM on chromosome 3H (for both years) (Figure 2B) and a region near VRS1 (80.5 cM) on $2 \mathrm{H}$ (Figure 2A). For the latter, in 2009 the most significant marker was MR_1435185P85F60 at $82.0 \mathrm{cM}$ while in 2010 the most significant marker was MR_48841P1435F22 at $83.2 \mathrm{cM}$. Regression analysis (in Genstat) was used to model the joint effects of these three locations on height. There were no significant interactions among the three QTLs, and so an additive regression on SNP MR_47526P1793R57 from 5H, SNP MR_1631678P7 82F7 from $3 \mathrm{H}$ and Vrs1/vrs1 on $2 \mathrm{H}$ (for consistency across years) was used. In 2009, these three locations jointly explained $76.6 \%$ of the variance in height. For MR_1631678P782F7 on 3H, the 'bb' Morex allele has a mean height 9.7 (se 1.7) cm higher than the 'aa' GP allele, and the vrs 1 types (six-row) on $2 \mathrm{H}$ had a mean height 10.1 (se 1.7) $\mathrm{cm}$ lower than the Vrs1 (two-row) types. In 2010, these three locations jointly explained $77.5 \%$ of the variance in height. For MR_1631678P782F7 (3H), the 'bb' allele has a mean height 8.9 (se 1.4) $\mathrm{cm}$ higher than the 'aa' allele and the vrs 1 types (six-row) $(2 \mathrm{H})$ have a mean height 6.8 (se 1.4) cm lower than the Vrs1 (two-row) types. The effect of excluding the two lines with discrepant heights in the two years was investigated, but the QTL locations were unchanged and the differences in the parameter estimates were negligible.

\section{What is the resolution of the GPMx GBS map at Ari-e.GP?}

To explore the potential of using GBS and the GPMx RIL population as a platform for gene identification, we used the barley genome assembly to determine the gene content surrounding Vrs1, Ari-e and the flanking GBS markers. As the Vrs1 gene is known [25] we investigated the interval containing the two GBS markers that cosegregated with Vrs1 and that fell between markers, MR_51408P1352R32 and MR_40453P5185R30. These defined a $1.5 \mathrm{cM}$ interval on the GPMx GBS map. Current information [21,22] indicates this interval corresponds to $4.13 \mathrm{Mb}$ on the barley physical map (Figure 3A). It contains an estimated 52 genes, resulting in a gene density estimate of 12.6 genes/Mb and defining this locus as gene rich (the genome-wide average gene density in barley is 5 genes/Mb).

Unlike VRS1, the identity of Ari-e is not known. The QTL peak for plant height in GPMx (i.e. the ari-e.GP locus) is associated with GBS marker MR_47526P17 93R57 which is flanked by MR_335403P1239R45 and MR_1560792P1192F41. These three markers define a $7.2 \mathrm{cM}$ interval on the GPMx GBS map. However on the barley physical map marker MR_137133P6361R37 (morex_contig_137133) appears to be positioned erroneously (Figure 3B) making it difficult to estimate the size of the relevant interval (Figure 3B). Replacing MR_13 7133P6361R37 with a distal marker, MR_137133P636 $1 \mathrm{R} 37$, the interval defined is $46 \mathrm{Mb}$ and contains an estimated 397 genes. We then investigated a more recent ordering of sequence contigs on the barley genome provided by the POPSEQ methodology [29]. There, the same region corresponds to a $3.3 \mathrm{cM}$ genetic interval in the Barke $\times$ Morex population and a $2.1 \mathrm{cM}$ interval in the Oregon Wolfe populations respectively. This region contains over 7,000 anchored sequence contigs spanning a total sequence length of approximately $10 \mathrm{Mb}$ [29]. As the contig sequences represent only a small portion of the physical sequence, Ari-e appears to be located in a relatively low recombining region. Despite residual recombination within this genetic interval, the lack of detected polymorphism suggests that the original parental haplotypes may be similar in this region and increased resolution will likely need to be sought in different populations.

\section{Conclusions}

We have shown that GBS is an effective approach for the generation of marker-dense genetic maps in cultivated barley. The short sequence tags enabled us to directly anchor the regions containing both VRS1 and ari-e.GP to the recently released integrated genetic and physical sequence assembly of the barley genome and to crudely define the physical size of the two genetic intervals that we investigated. Our hope was that the flanking markers would ultimately assist us in identifying ari-e.GP. Given the resolution we obtained around ari-e.GP this seems unlikely. Our data also indicate that a region encompassing the major morphological gene VRS1, which determines row-type and number of tillers in barley on $2 \mathrm{H}$ and an unknown locus on $3 \mathrm{H}$, also affect plant height in the GPMx population.

An important practical outcome of this work for us was that we found the GBS data more challenging to handle and subsequently to analyse than the current multiplex SNP assay technology we routinely run in the lab [9]. Indeed, this may discourage some groups from adopting the GBS approach. Nevertheless, as the principal determinant of resolution in genetic studies is a combination of the 

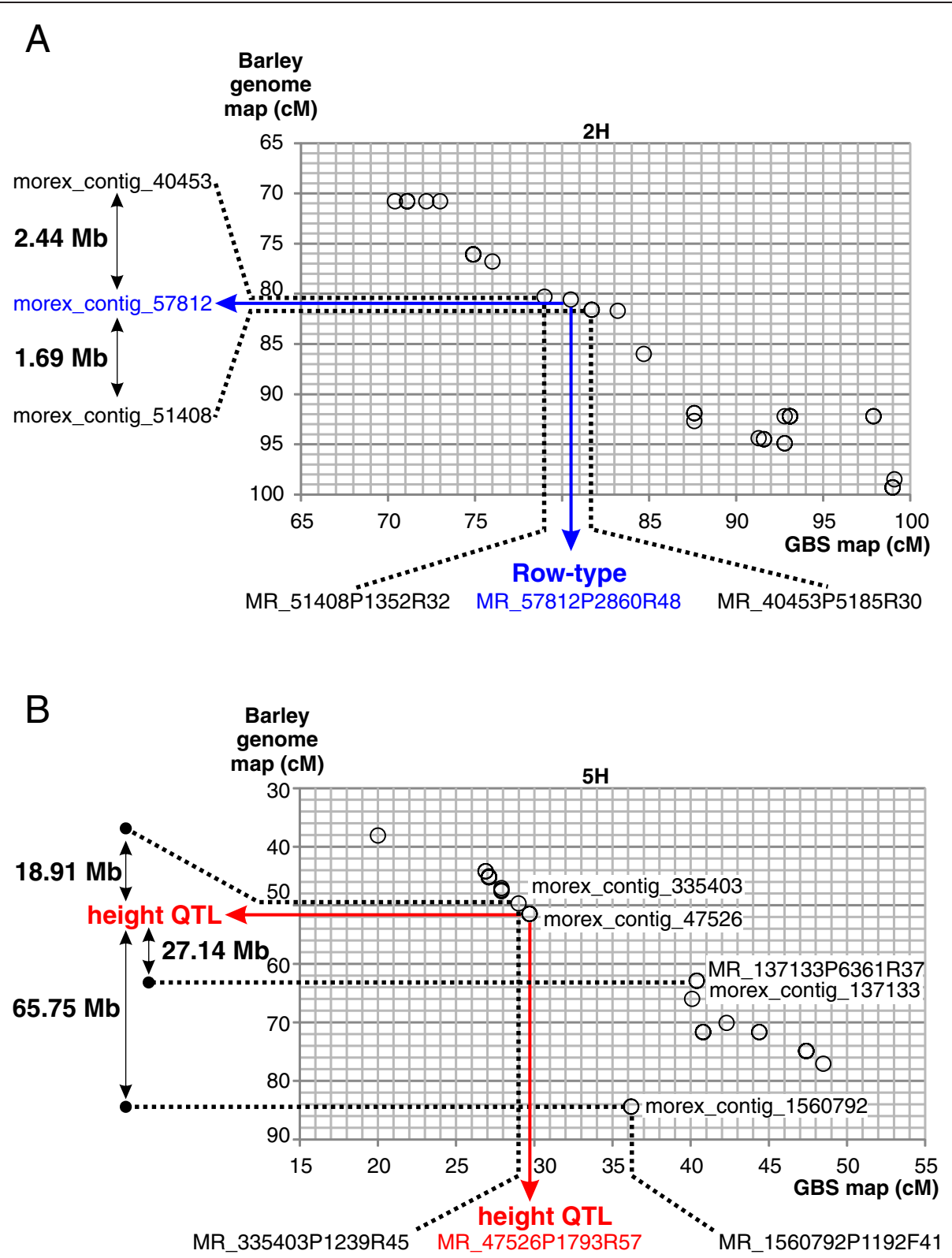

Figure 3 Scatterplots showing genetic positions of the markers on the GPMx GBS map versus genetic positions of corresponding markers on the barley genome map at the Vrs1 (panel A) and Ari-e (panel B) loci. Dotted lines highlight markers that flank these loci on the GPMx map. The map position of VRS1 and its co-segregating markers are shown in panel $\mathbf{A}$ in blue. Similarly, the position of the plant height QTL peak and the best marker associated with it is shown on the panel $\mathbf{B}$ in red. Physical distances between flanking markers and trait loci have been derived from the barley physical map presented in [22].

number of recombinants in the population and the number of genetic markers assayed, we were somewhat surprised (and disappointed) that with approximately 1,400 informative genetic markers covering a map length of $1,200 \mathrm{cM}$, the most closely linked markers to ari-e.GP spanned a region of approximately $7 \mathrm{cM}$. It is possible that the mutant ari-e.GP locus was induced within a local haplotype that is shared with Morex (i.e. the gene lies within a region of identity by descent or state between both parents). Indeed, evidence from the barley $9 \mathrm{~K}$ iSelect SNP genotyping platform on the parents indicates that GP and Morex probably do share a common haplotype across the proximal short arm and centromeric region of $5 \mathrm{H}$. This would unfortunately result in a markerless gap in the genetic map. Similar gaps have been evident in other highdensity genetic maps of barley $[9,12,15]$. Despite this, the flanking SNP-containing GBS tags will be easy to convert to single-locus markers, and these will be highly valuable 
for identifying additional recombinants around ari-e.GP and, if pursued further using a map based approach, ultimately the identification of the gene.

\section{Methods}

\section{Plant material and DNA samples}

The GPMx population was developed from a cross between a two-rowed barley (Golden Promise, ari-e.GP/ Vrs1) and six-rowed barley (Morex, Ari-e/vrs1) at the James Hutton Institute (JHI). DNAs were extracted from one week old seedling tissue using the DNeasy Plant Mini kit (Qiagen). Three 48-plex GBS libraries were constructed from a set of 138 progenies from the $\mathrm{F}_{11}$ single-seed descent generation, along with replicated samples of each parent, respectively.

\section{Plant growth and phenotyping}

Ten seeds harvested from a single $\mathrm{F}_{9}$ generation plant of the GPMx RIL population were planted in soil in a polytunnel in spring 2009. Planting was randomized and plants grown using automatic watering. Plant height measurements were performed on mature plants prior to harvest. Plant height for each line was determined by selecting the 3-5 longest tillers and measuring the distance from the ground to the top spikelets (excluding awns). Bulked seeds harvested from 3-5 plants of each line of the $F_{10}$ generation of the GPMx RIL population were planted in the field in spring 2010. Before planting, TGW (Thousand Grain Weight) of each sample was determined and used to calculate the weight of the seeds to be planted in $1 \times 2.5 \mathrm{~m}$ plots (so that each plot has about the same number of seeds). In total, 17 randomly selected lines and parents were planted as randomized replicates (2-3X). Plant height measurements were performed 3-4 weeks after anthesis following the same procedure as above. Lodged plants were lifted before measuring their height.

\section{Constructing GBS libraries}

GBS libraries were constructed in a similar manner to Poland et al. [15]. Briefly:

A set of 48 barcoded adapters (Additional file 1: Table S1) were generated from complementary oligonucleotides (Sigma) with a Pst I overhang sequence and unique barcodes of length $4 \mathrm{nt}$ to $8 \mathrm{nt}$. In addition, a common Y-adapter was generated corresponding to the 5' TA overhang generated by MseI. Top and bottom strand complementary oligonucleotides for each adapter $(50 \mu \mathrm{M})$ were annealed using the following program: $95^{\circ} \mathrm{C}$ for $2 \mathrm{~min}$, decrease to $25^{\circ} \mathrm{C}$ by $0.1^{\circ} \mathrm{C} / \mathrm{s}$, hold at $25^{\circ} \mathrm{C}$ for $30 \mathrm{~min}$. Annealed adapters were diluted 1:10 and their concentration measured using PicoGreen. Barcoded adapters were normalised to $2 \mathrm{ng} / \mu \mathrm{l}$ and the common Y-adapter to $40 \mathrm{ng} / \mu \mathrm{l}$.
DNAs were digested in $30 \mu \mathrm{l}$ reactions containing $200 \mathrm{ng}$ of genomic DNA, $1 \times$ NEB buffer 4, 8 u PstI-HF, $8 \mathrm{u} \mathrm{MseI}$, incubated at $37^{\circ} \mathrm{C}$ for $3 \mathrm{~h}$, then $80^{\circ} \mathrm{C}$ for $20 \mathrm{~min}$ to inactivate the enzymes. For ligation, $4 \mathrm{ng}$ annealed barcoded adapter and $200 \mathrm{ng}$ annealed common Y-adapter were added along with $1 \times$ T4 DNA ligase buffer and $200 \mathrm{u}$ T4 ligase in a total volume of $50 \mu \mathrm{l}$. All 48 ligation reactions were incubated at $22^{\circ} \mathrm{C}$ for $2 \mathrm{~h}$, then $65^{\circ} \mathrm{C}$ for $20 \mathrm{~min}$.

An aliquot $(5 \mu \mathrm{l})$ was removed from each ligation reaction, pooled, purified using QIAquick PCR Purification Kit (Qiagen) and eluted in $30 \mu \mathrm{l}$ of $\mathrm{dH}_{2} \mathrm{O}$. PCR amplification was conducted in $50 \mu \mathrm{l}$ reactions containing $4 \mu \mathrm{l}$ of pooled and purified library DNA, $1 \times$ high fidelity Phusion polymerase buffer, $0.2 \mu \mathrm{M}$ dNTP, $0.2 \mu \mathrm{M}$ primer 1 (complementary to barcode adapter), $0.2 \mu \mathrm{M}$ primer 2 (complementary to common Y-adapter), $1 \mathrm{u}$ Phusion polymerase Taq. PCR was conducted as follows: $98^{\circ} \mathrm{C}$ for $30 \mathrm{~s}$ for one cycle; 20 cycles of $98^{\circ} \mathrm{C}$ for $10 \mathrm{~s}, 65^{\circ} \mathrm{C}$ for $20 \mathrm{~s}, 68^{\circ} \mathrm{C}$ for $20 \mathrm{~s}$; one cycle of $75^{\circ} \mathrm{C}$ for $5 \mathrm{~min}$, cool to $4^{\circ} \mathrm{C}$. The PCR enriched library was gel-purified, selecting the 200-500 bp size fraction, using the MinElute Gel Extraction Kit (Qiagen), eluted in $12 \mu \mathrm{l} \mathrm{dH} 2 \mathrm{O}$, and quality and quantity of the library measured using a Nanodrop and Agilent Bioanalyzer.

\section{Sequencing and processing raw GBS data}

Single-end sequencing from the PstI sites was carried out using Illumina GA II and/or HiSeq2000 sequencer: of the three GBS libraries (GPMx_1, GpMx_2 \& GPMx_3), initially GPMx_1 was sequenced on two lanes of Illumina GAII and subsequently all three GBS libraries were sequenced on one lane each of Illumina HiSeq2000. All GBS sequences were submitted to Sequence Read Archive section of the European Nucleotide Archive (ENA) (submission: ERP002594 Genotyping by sequencing of a barley mapping population).

\section{Generation of reference sequences}

Reference sequences for the mapping of GBS tags were generated from existing genomic assemblies of the barley cultivars Morex, Bowman and Barke based on Illumina whole genome shotgun sequencing. As a first step in the workflow (see Additional file 3: Figure S1 for a diagram of the full workflow), the EMBOSS program restrict (http://emboss.sourceforge.net/) was used to discover PstI restriction sites in the assemblies. Custom written Java code was then used to extract from the Morex genomic assembly two separate flanking $64 \mathrm{bp}$ sequences extending the restriction site in forward and in reverse direction. This process was repeated for the other two cultivar assemblies and the extracted 64 bp sequences were then compared with the sequences generated from cultivar Morex assembly using the standalone 
BLASTN program [30] from NCBI (version 2.2.26+). A single hit was obtained per query, and from this we extracted those hits with alignments along the full length of the query sequence, an identity value of less than $100 \%$, and a mismatch number of at least 2 . These hits were added to the full set of Morex flanking sequences, thereby providing a global set of reference sequences from the three barley genome assemblies. To further refine the reference sequences, we screened them for chloroplast DNA, which can be a common feature in whole genome shotgun sequencing. This was done by BLASTN, with the combined set of sequences as query against the full barley chloroplast genome sequence (http://www.ncbi.nlm. nih.gov/nuccore/118430366?report=fasta). Hits were filtered to require sequence identity $>=90 \%$, and an alignment length $>=64$. We detected 568 chloroplast DNA sequences that were subsequently removed from the reference set.

\section{Read mapping}

Prior to mapping, the raw Illumina reads were assigned to their respective samples ('deconvoluted') based on the sample-specific barcodes included in the sequence. Barcode lengths varied between 4 and 8 bases therefore custom written Java code was used for deconvolution, and this also removed the barcodes after assigning the read to a sample, which is a requirement for the successful mapping of the read to a reference sequence. Reads that started with the PstI overhang sequence (TGCAG) after barcode removal were accepted, quality trimmed to remove bases of quality Phred $<20$ from the 3 '-end (distal to the PstI site), and then shortened from the 3'-end to a standard length of 64 bases. Reads that were shorter than this after quality trimming were discarded.

Reads were then mapped to the 64 bp reference sequences using the Bowtie mapping tool (version 0.12.7, [24]). To avoid cross-mapping of reads between similar sequences, the "-best -strata" switch was used, which ensures that multi-mapped reads are only mapped to the location with the fewest mismatches. In order to reduce the number of false positive SNPs during downstream analysis, only a single mismatch per read was allowed (“-v 1"), and only uniquely mapped reads were retained (“-m 1").

\section{SNP discovery and genotype calling}

We used the FreeBayes software [31] to discover single nucleotide polymorphisms (SNPs), as well as custom Java code for converting the resulting VCF file into a human-readable text file. Within FreeBayes, the SNPs were filtered to retain those where the minimum number of reads with the alternative allele was greater than 3 , which provided a total of 57,328 SNPs. We then applied the following filters: the minimum fraction of reads with the alternative allele for a SNP should be greater than or equal to 0.1 ; the percentage difference between the base qualities for the reference and alternative alleles should be less than or equal to 5; the SNP quality score cut-off should be greater than or equal to 20. This procedure yielded 18,251 SNPs. Then, within Excel, further filters were applied: we required a total read coverage of greater than or equal to 700 (ie. a mean of at least 5 reads for each sample in the population), which left 3,246 SNPs; the percentage of heterozygous samples was less than or equal to $2 \%$, which left 1,985 SNPs; the ratio of alternative allele/reference allele was greater than or equal to 0.5 , which left 1,968 SNPs.

Genotypes were then called based on the proportion of the reference allele. We identified this as homozygous for the reference allele if the proportion was greater than 0.8 , as homozygous for the alternative allele if the proportion was less than 0.2 and as heterozygous if the proportion is between 0.2 and 0.8 . Samples with fewer than three reads if designated homozygous, or with fewer than six reads if designated heterozygous, were recoded as missing. Nineteen SNPs had a missing genotype for one of the parents, and these were also excluded to leave 1,949 SNPs for linkage mapping. Visual inspection of both mappings and SNPs was carried out using the Tablet software [32].

\section{Linkage mapping}

The SNP data were sorted by decreasing quality score before analysis with JoinMap [33]. This ensured that when co-segregating SNPs were excluded, the lower quality SNPs were preferentially dropped. SNPs with greater than $20 \%$ missing values were also excluded from the JoinMap analysis. SNPs were grouped using the independence LOD score, and then ordered within each linkage group using the maximum likelihood algorithm. The GBS tags were mapped to reference sequences generated from Morex, Bowman and Barke WGS shotgun assemblies. Those from Morex contain previously published anchored genetic/ physical markers, which we assumed to be correct. We define these as anchoring markers on the genetic linkage groups. Additional file 4: Table S3 provides a list of 1,332 unique co-dominant GBS markers used for map construction and ordered according to their map location on the GPMx population. It highlights 403 genetically redundant markers, the correspondence of all GBS tags to expressed genes (MLOC's) and their genetic position on the IBSC consensus map (IBSC, 2012).

\section{QTL mapping}

QTL interval mapping was used to locate QTLs for the 2009 and 2010 height data separately, using MapQTL [34]. A permutation test with 1,000 permutations was used to establish the LOD threshold. Restricted multiple 
QTL mapping (rMQM mapping) was used to search for further QTLs taking into account the most significant ones. A regression analysis, using Genstat 15 for Windows [35], was used to test for significant interactions among the selected QTLs. Genstat was also used to test which of the mapped SNPs showed the greatest association with the two-rowed/six-rowed type, using chisquare tests of independence.

\section{Cross-referencing barley genome data sets}

In total 4,607 individual sequences from the manifest files accompanying barley OPA SNP mapping platforms [36] were used to identify corresponding sequences in the barley genome represented by $\sim 2.6$ million sequence contigs using the blastN algorithm [9]. The resulting table cross-referenced SNP markers used to define introgressions in the Bowman backcross derived lines [28] and sequence contigs in the barley genome assembly [22]. Tables containing the contig anchoring results from POPSEQ [29] are available for download from ftp://ftp. ipk-gatersleben.de/barley-popseq/.

\section{Additional files}

Additional file 1: Table S1. Barcode adapters used for GBS.

Additional file 2: Table S2. GBS read distribution.

Additional file 3: Figure S1. Data processing workflow for the raw GBS Illumina reads.

Additional file 4: Table S3. Co-dominant GBS markers.

\section{Competing interests}

The authors declare that they have no competing interests.

\section{Authors' contributions}

$\mathrm{HL}, \mathrm{PH}, \mathrm{AD}$ and RW conceived the study. $\mathrm{HL}$ and $\mathrm{PH}$ established and conducted the GBS analysis of the GPMx population with guidance from JP. $A D$ developed and phenotyped the GPMx population. $H L, J R, A D, L R$ and $C H$ conducted statistical analysis of the genetic data with all NGS data processing steps conducted by $\mathrm{MB}$ and $\mathrm{HL}$. HL, AD, PH, MB, CH and RW wrote the manuscript, and all authors read and approved the final version. RW and $\mathrm{PH}$ supervised the work and obtained the funding that allowed the study to be conducted.

\section{Acknowledgements}

We would like to acknowledge Jill Alexander, Nicola Bonar, Richard Keith, Pauline Smith and Ilze Druka for excellent technical assistance, in particular during population development and phenotypic analysis. The majority of the funding for the described research came from the EU FP7 project 'TriticeaeGenome' and the Scottish Government's Rural and Environment Science and Analytical Services Division (RESAS) Strategic Research Programme Workpackage 5.2.

\section{Author details}

${ }^{1}$ Cell and Molecular Sciences, The James Hutton Institute, Invergowrie, Dundee, Scotland DD2 5DA, UK. ${ }^{2}$ Biomathematics and Statistics Scotland (BioSS), Invergowrie, Dundee, Scotland DD2 5DA, UK. ${ }^{3}$ Hard Winter Wheat Genetics Research Unit, USDA-ARS and Department of Agronomy, Kansas State University, 4011 Throckmorton, Manhattan, KS 66506, USA. ${ }^{4}$ Biomedical Sciences Research Complex, University of St Andrews, North Haugh, St Andrews, Scotland KY16 9ST, UK. ${ }^{5}$ Division of Plant Sciences, James Hutton Institute, Invergowrie, The University of Dundee. College of Life Sciences, Dundee, Scotland DD2 5DA, UK.
Received: 31 October 2013 Accepted: 3 February 2014

Published: 6 February 2014

\section{References}

1. Ellis RP, Forster BP, Gordon DC, Handley LL, Keith RP, Lawrence P, Meyer R, Powell W, Robinson D, Scrimgeour CM, et al: Phenotype/genotype associations for yield and salt tolerance in a barley mapping population segregating for two dwarfing genes. J Exp Bot 2002, 53(371):1163-1176.

2. Forster BP: Mutation genetics of salt tolerance in barley: an assessment of Golden Promise and other semi-dwarf mutants. Euphytica 1994, 120(3):317-328.

3. Malosetti M, van Eeuwijk FA, Boer MP, Casas AM, Elia M, Moralejo M, Bhat PR, Ramsay L, Molina-Cano JL: Gene and QTL detection in a three-way barley cross under selection by a mixed model with kinship information using SNPs. Theor Appl Genet 2011, 122(8):1605-1616.

4. Siedler H, Graner A: Construction of physical maps of the Hor1 locus of two barley cultivars by pulsed field gel electrophoresis. Mol Gen Genet 1991, 226(1-2):177-181.

5. Waugh R, Bonar N, Baird E, Thomas B, Graner A, Hayes PM, Powell W: Homology of AFLP products in three mapping populations of barley. Mol Gen Genet 1997, 255(3):311-321.

6. Ramsay L, Macaulay M, Ivanissevich SD, MacLean K, Cardle L, Fuller JD, Edwards KJ, Tuvesson S, Morgante M, Massari A, Maestri E, Marmiroli N, Sjakste T, Ganal M, Powell W, Waugh R: A simple sequence repeat-based linkage map of barley. Genetics 2000, 156:1997-2005.

7. Wenzl P, Carling J, Kudrna D, Jaccoud D, Huttner E, Kleinhofs A, Kilian A: Diversity Arrays Technology (DArT) for whole-genome profiling of barley. Proc Natl Acad Sci USA 2004, 101(26):9915-9920.

8. Rostoks N, Mudie S, Cardle L, Russell J, Ramsay L, Booth A, Svensson JT, Wanamaker SI, Walia H, Rodriguez EM, et al: Genome-wide SNP discovery and linkage analysis in barley based on genes responsive to abiotic stress. Mol Genet Genomics 2005, 274(5):515-527.

9. Comadran J, Kilian B, Russell J, Ramsay L, Stein N, Ganal M, Shaw P, Bayer M, Thomas W, Marshall D, et al: Natural variation in a homolog of Antirrhinum CENTRORADIALIS contributed to spring growth habit and environmental adaptation in cultivated barley. Nat Genet 2012, 44(12):1388-1392.

10. Moragues M, Comadran J, Waugh R, Milne I, Flavell AJ, Russell JR: Effects of ascertainment bias and marker number on estimations of barley diversity from high-throughput SNP genotype data. Theor App/ Genet 2010, 120(8):1525-1534.

11. Baird NA, Etter PD, Atwood TS, Currey MC, Shiver AL, Lewis ZA, Selker EU, Cresko WA, Johnson EA: Rapid SNP discovery and genetic mapping using sequenced RAD markers. PLOS One 2008, 3(10):e3376.

12. Chutimanitsakun Y, Nipper RW, Cuesta-Marcos A, Cistue L, Corey A, Filichkina T, Johnson EA, Hayes PM: Construction and application for QTL analysis of a Restriction Site Associated DNA (RAD) linkage map in barley. BMC Genomics 2011, 12:4.

13. Elshire RJ, Glaubitz JC, Sun Q, Poland JA, Kawamoto K, Buckler ES, Mitchell SE: A robust, simple genotyping-by-sequencing (GBS) approach for high diversity species. PLoS One 2011, 6(5):e19379.

14. McMullen MD, Kresovich S, Villeda HS, Bradbury P, Li H, Sun Q, Flint-Garcia S, Thornsberry J, Acharya C, Bottoms C, et al: Genetic properties of the maize nested association mapping population. Science 2009, 325(5941):737-740.

15. Poland JA, Brown PJ, Sorrells ME, Jannink JL: Development of high-density genetic maps for barley and wheat using a novel two-enzyme genotyping-by-sequencing approach. PLoS One 2012, 7(2):e32253.

16. Bus A, Hecht J, Huettel B, Reinhardt R, Stich B: High-throughput polymorphism detection and genotyping in Brassica napus using next-generation RAD sequencing. BMC Genomics 2012, 13:281.

17. Yang H, Tao Y, Zheng Z, Li C, Sweetingham MW, Howieson JG: Application of next-generation sequencing for rapid marker development in molecular plant breeding: a case study on anthracnose disease resistance in Lupinus angustifolius L. BMC Genomics 2012, 13:318.

18. Truong HT, Ramos AM, Yalcin F, de Ruiter M, van der Poel HJ, Huvenaars $\mathrm{KH}$, Hogers RC, van Enckevort $L$, Janssen A, van Orsouw NJ, et al: Sequence-based genotyping for marker discovery and co-dominant scoring in germplasm and populations. PLoS One 2012, 7(5):e37565.

19. Lu F, Lipka AE, Glaubitz J, Elshire R, Cherney JH, et al: Switchgrass genomic diversity, ploidy, and evolution: novel insights from a network-based SNP discovery protocol. PLoS genetics 2013, 9:e1003215. 
20. Sonah H, Bastien M, lquira E, Tardivel A, Légaré G, et al: An improved genotyping by sequencing (GBS) approach offering increased versatility and efficiency of SNP discovery and genotyping. PloS One 2013, 8:e54603.

21. Mayer KF, Martis M, Hedley PE, Simkova H, Liu H, Morris JA, Steuernagel B, Taudien S, Roessner S, Gundlach H, et al: Unlocking the barley genome by chromosomal and comparative genomics. Plant Cell 2011, 23(4):1249-1263.

22. Mayer KF, Waugh R, Brown JW, Schulman A, Langridge P, Platzer M, Fincher GB, Muehlbauer GJ, Sato K, Close TJ, et al: A physical, genetic and functional sequence assembly of the barley genome. Nature 2012, 491(7426):711-716.

23. Mayer KF, Taudien S, Martis M, Simková H, Suchánková P, Gundlach H, Wicker T, Petzold A, Felder M, Steuernagel B, Scholz U, Graner A, Platzer M, Dolezel J, Stein N: Gene content and virtual gene order of barley chromosome 1H. Plant Physiol 2009, 151(2):496-505.

24. Langmead B, Schatz MC, Lin J, Pop M, Salzberg SL: Searching for SNPs with cloud computing. Genome Biol 2009, 10(11):R134.

25. Komatsuda T, Pourkheirandish M, He C, Azhaguvel P, Kanamori H, Perovic D, Stein N, Graner A, Wicker T, Tagiri A, et al: Six-rowed barley originated from a mutation in a homeodomain-leucine zipper I-class homeobox gene. Proc Natl Acad Sci USA 2007, 104(4):1424-1429.

26. Kucera J, Lundqvist U, Gustafsson A: Induction of breviaristatum mutants in barley. Hereditas 1975, 80(2):263-278.

27. Thomas WTB, Powell W, Wood W: The chromosomal location of the dwarfing gene present in the spring barley variety golden promise. Heredity 1984, 53:177-183.

28. Druka A, Franckowiak J, Lundqvist U, Bonar N, Alexander J, Houston K, Radovic S, Shahinnia F, Vendramin V, Morgante M, et al: Genetic dissection of barley morphology and development. Plant Physiol 2011, 155(2):617-627.

29. Mascher M, Munoz-Amatriain M, Barry K, Close TJ, Himmelback A, Poland J, Mayer KFX, Rokhsar D, Schmutz J, Scholz U, Schulman AH, Wise RP, Muehlbauer GJ, Stein N, Waugh R: Anchoring and ordering NGS contig assemblies by population sequencing (POPSEQ). Plant $J 2013$. doi: 10.1111/tpj.12319.

30. Altschul SF, Gish W, Miller W, Myers EW, Lipman DJ: Basic local alignment search tool. J Mol Biol 1990, 215(3):403-410.

31. Garrison E: FreeBayes. In Marth Lab. Boston, US: Boston College; 2012. http://bioinformatics.bc.edu/marthlab.

32. Milne I, Stephen G, Bayer M, Cock PJA, Pritchard L, Cardle L, Shaw PD, Marshall D: Using tablet for visual exploration of second-generation sequencing data. Brief Bioinform 2013, 14(2):193-202.

33. Van Ooijen JW: JoinMap ${ }^{\star} 4$, Software for the Calculation of Genetic Linkage Maps in Experimental Populations. Wageningen: Kyazma BV; 2006.

34. Van Ooijen JW: MapQTL ${ }^{\circledR}$ 5, Software for the Mapping of Quantitative Trait Loci in Experimental Populations. Wageningen, the Netherlands: Kyazma BV; 2004.

35. Payne R, Murray D, Harding S, Baird D, Soutar D: Introduction to Genstat for Windows. 15th edition. Hemel Hempstead: VSN International; 2012.

36. Close TJ, Bhat PR, Lonardi S, Wu Y, Rostoks N, Ramsay L, Druka A, Stein N, Svensson JT, Wanamaker $S$, et al: Development and implementation of high-throughput SNP genotyping in barley. BMC Genomics 2009, 10:582.

\section{Submit your next manuscript to BioMed Central and take full advantage of:}

- Convenient online submission

- Thorough peer review

- No space constraints or color figure charges

- Immediate publication on acceptance

- Inclusion in PubMed, CAS, Scopus and Google Scholar

- Research which is freely available for redistribution

Submit your manuscript at www.biomedcentral.com/submit
C Biomed Central 